\title{
Dynamic Modeling from Flight Data with Unknown Time Skews
}

\author{
Eugene A. Morelli * \\ NASA Langley Research Center, Hampton, Virginia, 23681
}

\begin{abstract}
A method for estimating dynamic model parameters from flight data with unknown time skews is described and demonstrated. The method combines data reconstruction, nonlinear optimization, and equation-error parameter estimation in the frequency domain to accurately estimate both dynamic model parameters and the relative time skews in the data. Data from a nonlinear F-16 aircraft simulation with realistic noise, instrumentation errors, and arbitrary time skews were used to demonstrate the approach. The approach was further evaluated using flight data from a subscale jet transport aircraft, where the measured data were known to have relative time skews. Comparison of modeling results obtained from time-skewed and time-synchronized data showed that the method accurately estimates both dynamic model parameters and relative time skew parameters from flight data with unknown time skews.
\end{abstract}

\section{Nomenclature}

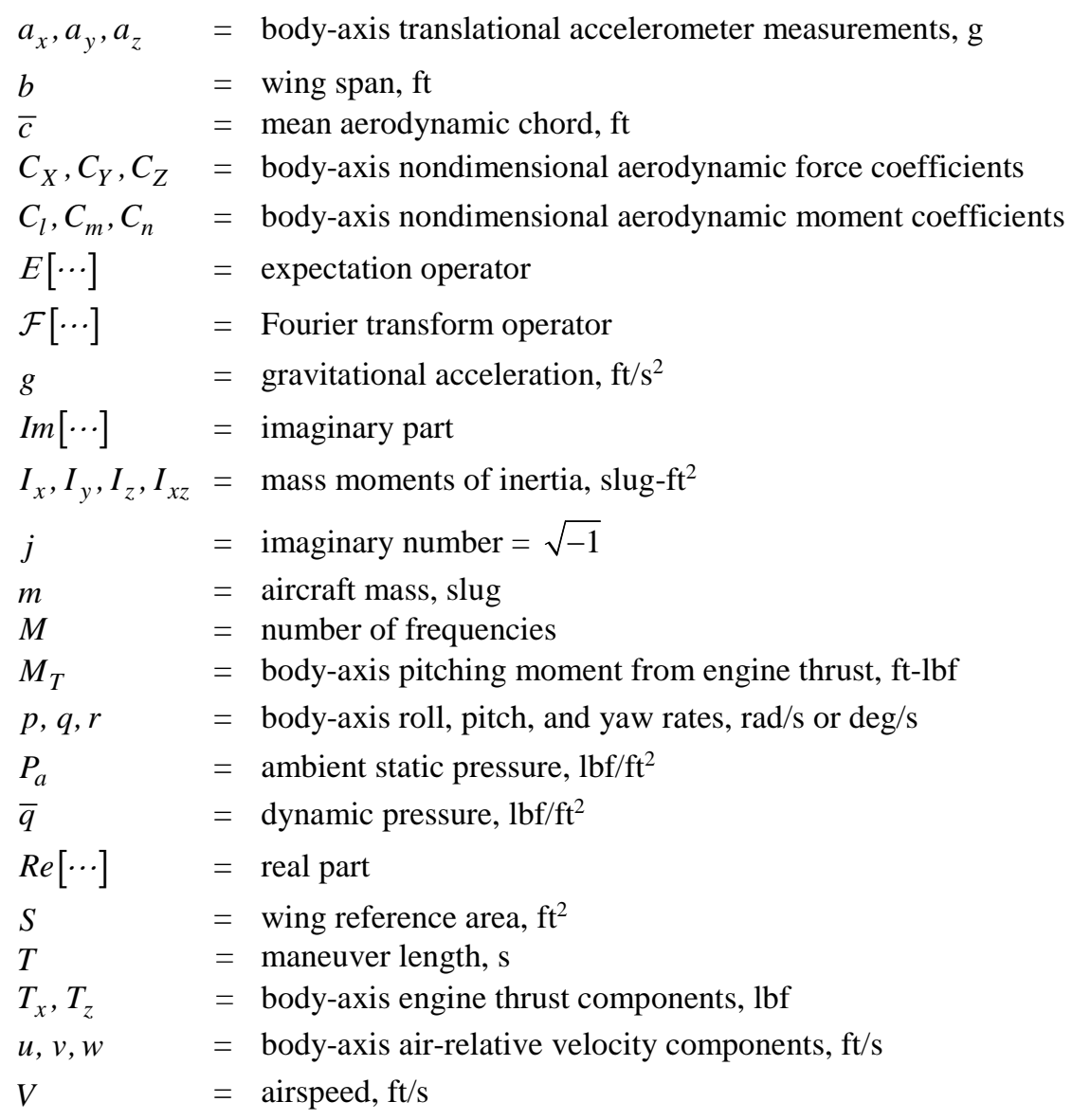

\footnotetext{
* Senior Research Engineer, Dynamic Systems and Control Branch, MS 308, Associate Fellow
} 


$\begin{array}{ll}\alpha & =\text { angle of attack, rad or deg } \\ \beta & =\text { sideslip angle, rad or deg } \\ \delta_{s}, \delta_{e}, \delta_{a}, \delta_{r} & =\text { stabilator, elevator, aileron, and rudder deflections, rad or deg } \\ \phi, \theta, \psi & =\text { Euler roll, pitch, and yaw angles, rad or deg } \\ \boldsymbol{\theta} & =\text { parameter vector } \\ \boldsymbol{\Sigma} & =\text { covariance matrix } \\ \underline{\text { superscripts }} & \\ T & \text { transpose } \\ - & \text { estimate } \\ \cdot & \text { time derivative } \\ \sim & \text { Fourier transform } \\ -1 & \text { matrix inverse } \\ \dagger & \text { complex conjugate transpose } \\ \underline{\text { subscripts }} & \\ c g & =\text { center of gravity } \\ I & =\text { inboard } \\ L & =\text { left } \\ r & =\text { reconstructed } \\ R & =\text { right } \\ o & =\text { reference value or base term } \\ O & =\text { outboard }\end{array}$

\section{Introduction}

G VERY commonly-used method for dynamic modeling based on measured data makes an implicit assumption Lthat all measured signals are sampled at the same time ${ }^{1,2}$. In practice, this is never exactly true, due to issues such as multiplexing in the analog-to-digital conversion electronics, and different sampling rates for various signals, for example. In high-quality aircraft instrumentation systems designed to collect data for dynamic modeling, such issues are well known, and steps are taken to minimize the differences in the sampling times for the measured data. The usual approaches involve accurately recording the time when each sample was taken (time tagging), and/or linear interpolation to approximate simultaneous data sampling.

Unfortunately, not every dynamic modeling task is undertaken with data that have been carefully time tagged or interpolated. Some examples are data from an aircraft accident, and data from a low-budget instrumentation system, such as might be found on munitions, unmanned air vehicles, or remotely-piloted vehicles. Past approaches to solving this problem have included estimating the time skews separately, as well as estimating model parameters and time skews simultaneously in a combined nonlinear parameter estimation formulation ${ }^{3-5}$. In some practical situations, the effort necessary to time synchronize the sampled data is prohibitively expensive, and sometimes not even possible.

This paper reports the results of an investigation focused on finding a dynamic modeling method that can be used in cases where the sampled signals may have unknown relative time skews. This can simply mean that the assumption of simultaneous data sampling has been violated, and the exact sampling times of the measured signals are not known. But it can also mean that there are practical or instrumentation issues that produce a time skew in the signal before it is sampled. Common examples are lags in air flow angle data due to sensor position on the aircraft or pressure tubing lengths, and control surface data time skews from low sampling rates or the use of control surface command data rather than control surface position data. For conventional dynamic modeling methods, the presence of relative time skews in the measured data has known detrimental effects on dynamic modeling accuracy ${ }^{6}$. 
A real-world example of the importance of this issue is the first flight of the X-43A hypersonic research vehicle, or Hyper-X, which was aborted during the launch phase ${ }^{7}$. Investigation of the event focused (among other things) on the dynamic model of the booster and Hyper-X stack (see Fig. 1) in the transonic region. Uncertainty in the sample times of the measured signals used in the investigation led to an extensive effort to accurately determine what those sample times were. It was very important to the mishap investigation to determine the sample times accurately, so that the dynamic modeling based on measured flight data would produce accurate results, and the root cause of the mishap could be identified. A dynamic modeling method that could be applied regardless of the actual relative time skews in the data would have saved a large amount of time and expense in the mishap investigation.

The purpose of this paper is to explain and demonstrate a method

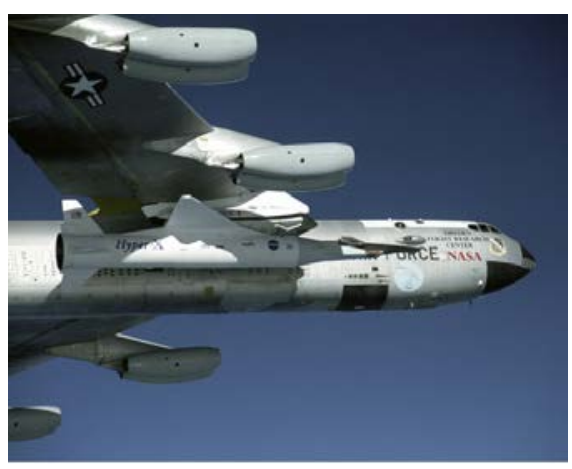

Figure 1. Hyper-X stack being carried to altitude on the NASA B-52B

Credit: NASA Armstrong Flight Research Center for accurately identifying dynamic models based on flight data with unknown time skews. The next section describes the general idea and gives some background information. Following this, the method is applied to data from an F-16 nonlinear simulation, using realistic noise, instrumentation errors, and known time skews in the data. Finally, flight data from a subscale transport aircraft were used to demonstrate that the method produces accurate modeling results for data with unknown time skews, and can also serve as a diagnostic tool to identify data that have significant relative time skews.

\section{Method}

In the time domain, it is difficult to accurately determine time skews in sampled data, because of the quantization related to the sampling interval and the fact that implementing any time skew value that is not an integer multiple of the sampling interval involves interpolation of the data. This causes convergence problems in time-domain parameter estimation algorithms, as well as resolution limitations for the estimated values of the time skews. However, if the analysis is done in the frequency domain, the time skews are continuous-valued parameters, and these problems do not exist. Consequently, the approach described here uses frequency-domain methods.

The problems that arise from time-skewed data are related to relative time skews, or time skews of sampled time series relative to one another. If all of the time series are time skewed in exactly the same way, then for dynamic modeling purposes, there is no problem, and the modeling can proceed normally. When the data have relative time skews, these time skews can be falsely interpreted as phase lag due to the system dynamics, for example, and this causes errors in the model parameter estimates.

A good approach to the problem is to first define a reference for the time skews in the data. Body-axis translational accelerations and angular rates typically come from a single Inertial Measurement Unit (IMU), so that the time skew between these measured quantities is negligible, and the data from the IMU provide a good reference for the time skews. Time skews for other sampled time series can be defined relative to this reference. With this timing reference established, there are typically two categories of data whose time skew must be determined relative to the reference:

1) Air flow data: airspeed $V$, sideslip angle $\beta$, and angle of attack $\alpha$

2) Control surface deflection data: elevator $\delta_{e}$, aileron $\delta_{a}$, and rudder $\delta_{r}$ (and possibly other controls)

For example, aircraft longitudinal short-period dynamics can be described by the following linear equations ${ }^{1}$ :

$$
\begin{gathered}
\dot{\alpha}\left(t-\tau_{\alpha}\right)=Z_{\alpha} \alpha\left(t-\tau_{\alpha}\right)+\left(1+Z_{q}\right) q(t)+Z_{\delta_{e}} \delta_{e}\left(t-\tau_{\delta_{e}}\right) \\
\dot{q}(t)=M_{\alpha} \alpha\left(t-\tau_{\alpha}\right)+M_{q} q(t)+M_{\delta_{e}} \delta_{e}\left(t-\tau_{\delta_{e}}\right)
\end{gathered}
$$




$$
a_{z}(t)=\frac{V_{o}}{g}\left[Z_{\alpha} \alpha\left(t-\tau_{\alpha}\right)+Z_{q} q(t)+Z_{\delta_{e}} \delta_{e}\left(t-\tau_{\delta_{e}}\right)\right]
$$

where the dimensional stability and control derivatives $Z_{\alpha}, Z_{q}, Z_{\delta_{e}}, M_{\alpha}, M_{q}, M_{\delta_{e}}$, and the relative time skews $\tau_{\alpha}, \tau_{\delta_{e}}$, are unknown parameters to be estimated from the data. The aircraft response and control variables in Eqs. (1) are perturbation quantities, as usual for linearized dynamic equations ${ }^{1}$.

Equations (1) can be readily transformed into the frequency domain ${ }^{1}$,

$$
\begin{gathered}
j \omega \tilde{\alpha} e^{-j \omega \tau_{\alpha}}=Z_{\alpha} \tilde{\alpha} e^{-j \omega \tau_{\alpha}}+\left(1+Z_{q}\right) \tilde{q}+Z_{\delta_{e}} \tilde{\delta}_{e} e^{-j \omega \tau_{\delta_{e}}} \\
j \omega \tilde{q}=M_{\alpha} \tilde{\alpha} e^{-j \omega \tau_{\alpha}}+M_{q} \tilde{q}+M_{\delta_{e}} \tilde{\delta}_{e} e^{-j \omega \tau_{\delta_{e}}} \\
\tilde{a}_{z}=\frac{V_{o}}{g}\left[Z_{\alpha} \tilde{\alpha} e^{-j \omega \tau_{\alpha}}+Z_{q} q+Z_{\delta_{e}} \tilde{\delta}_{e} e^{-j \omega \tau_{\delta_{e}}}\right]
\end{gathered}
$$

In the transformed Eqs. (2), the relative time skews are continuous-valued parameters that can be estimated in the same manner as the stability and control derivatives. At first glance, it would appear that all of the unknown parameters in Eqs. (2) could be estimated from the data using standard equation-error or output-error parameter estimation in the frequency domain ${ }^{1,8}$. However, this is not true, because the relative time skew parameter for angle of attack is highly correlated with the stability derivatives, which leads to inaccurate parameter estimates. The reason for this can be understood physically - any time skew in the angle of attack data can be interpreted as phase shift in the dynamic system, which can be modeled nearly equally well by adjusting the values of the stability derivatives or by adjusting the angle of attack time skew parameter. This is called model parameter correlation, which gives rise to an ill-posed parameter estimation problem that cannot be solved accurately. Note that the control time skew parameter does not have this problem, because the control has nothing to do with the phase shift associated with the dynamic system. Consequently, control time skew parameters are well-conditioned, identifiable parameters that can be estimated accurately along with the stability and control derivatives, using standard parameter estimation algorithms in the frequency domain.

The correlation between the stability derivatives and the angle of attack time skew can be broken if an accurate independent estimate of the angle of attack time skew parameter can be found. This can be done using data reconstruction, which in some contexts is called flight path reconstruction. In this approach, air flow data are reconstructed using IMU data (defined as having zero time skew) for translational accelerations, angular rates, and Euler attitude angles. The reconstructed air flow data are transformed into the frequency domain, as explained in the next subsection. Then a simple nonlinear optimization in the frequency domain can be used to accurately determine the relative time skews for the air flow data (airspeed, sideslip angle, and angle of attack). Once these relative time skew quantities are estimated accurately and separately, the resulting values are substituted into the dynamic equations (e.g., Eqs. (2)), and the remaining unknown parameters, consisting of stability and control derivatives and control time skew parameters, can be estimated accurately using a nonlinear parameter estimation algorithm in the frequency domain.

The next section describes transformation of measured flight data into the frequency domain. This is followed by an explanation of the data reconstruction and nonlinear optimization in the frequency domain used to accurately estimate time skews in the air-flow data. Then, the application of parameter estimation in the frequency domain to estimate stability and control parameters and the control time skews is explained, which completes the modeling. Because all of the unknown model parameters are estimated using standard parameter estimation algorithms in the frequency domain, the uncertainties in the estimated parameters can also be computed using standard methods ${ }^{1}$. 


\section{A. Transforming Data from the Time Domain into the Frequency Domain}

Measured flight data are transformed from the time domain into the frequency domain using the finite Fourier transform. For an arbitrary scalar signal $x(t)$ on the time interval $[0, T]$, the finite Fourier transform is defined by

$$
\mathcal{F}[x(t)] \equiv \tilde{x}(\omega) \equiv \int_{0}^{T} x(t) e^{-j \omega t} d t
$$

The finite Fourier transform can be computed very accurately for arbitrary frequencies $\omega$ using a numerical method based on Filon quadrature and cubic interpolation ${ }^{1,9}$.

When a time series has non-zero endpoints, the finite Fourier transform of the time derivative of that time series requires endpoint correction terms. Applying integration by parts to Eq. (3),

$$
\begin{aligned}
\mathcal{F}[\dot{x}(t)] & \equiv \int_{0}^{T} \dot{x}(t) e^{-j \omega t} d t \\
& =x(T) e^{-j \omega T}-x(0)+j \omega \int_{0}^{T} x(t) e^{-j \omega t} d t \\
& =x(T) e^{-j \omega T}-x(0)+j \omega \tilde{x}(\omega)
\end{aligned}
$$

Applying high-pass filtering to a time series or assuming perturbations from an initial reference condition imposes a zero initial condition. This leaves only one term for the endpoint corrections, so that

$$
\mathcal{F}[\dot{x}(t)]=x(T) e^{-j \omega T}+j \omega \tilde{x}(\omega)
$$

Equation (5) was used to compute the finite Fourier transform for the time derivative of a time series that has been high-pass filtered or conditioned as a perturbation from an initial reference condition.

\section{B. Data Reconstruction and Time Skew Estimation in the Frequency Domain}

Data from the IMU can be used in the nonlinear translational kinematic equations of motion to reconstruct body-axis velocity components $u, v, w$, from which $V, \beta, \alpha$ can be computed. The required equations are ${ }^{1}$ :

$$
\begin{gathered}
\dot{u}=r v-q w-g \sin \theta+g a_{x} \\
\dot{v}=p w-r u+g \cos \theta \sin \phi+g a_{y} \\
\dot{w}=q u-p v+g \cos \theta \cos \phi+g a_{z}
\end{gathered}
$$

Airspeed, sideslip angle, and angle of attack can be computed from $u, v$, and $w$ using ${ }^{1}$

$$
\begin{gathered}
V_{r}=\sqrt{u^{2}+v^{2}+w^{2}} \\
\beta_{r}=\sin ^{-1}\left(v / V_{r}\right) \\
\alpha_{r}=\tan ^{-1}(w / u)
\end{gathered}
$$

Equations (6)-(7) can be used to reconstruct airspeed, sideslip angle, and angle of attack data using IMU measurements. Consequently, the reconstructed $V_{r}, \beta_{r}, \alpha_{r}$ time series do not have relative time skew. 
A practical problem with this data reconstruction is that IMU data for angular rates $p, q, r$, and translational accelerations $a_{x}, a_{y}, a_{z}$, all typically have bias errors. This causes a time-dependent drift in the $V_{r}, \beta_{r}, \alpha_{r}$ reconstructions from Eqs. (6)-(7), even when the bias errors are small, because of the additive effect of the time integration. Furthermore, the initial conditions for the integrations in Eqs. (6) must be specified, and any errors in these values will bias the reconstructed data. Scale factor errors for angular rate and translational accelerometer measurements are negligible in practice, and any errors in the Euler angles $\phi$ and $\theta$ will be mitigated by the trigonometric functions. Most IMU provide Euler angle time series (or equivalent quaternion data) that have been corrected for drift, using magnetometer measurements, for example.

For the data reconstruction in Eqs. (6), initial conditions must be specified. The initial conditions can be computed from initial measured values for the air flow data, or a mean of measured values in a steady initial flight condition. Initial conditions for the body-axis velocity component reconstructions are then computed from

$$
\begin{gathered}
u(0)=V(0) \cos [\alpha(0)] \cos [\beta(0)] \\
v(0)=V(0) \sin [\alpha(0)] \\
w(0)=V(0) \sin [\alpha(0)] \cos [\beta(0)]
\end{gathered}
$$

In practical situations, particularly when the initial flight condition is not steady, this approach to finding the initial conditions for Eqs. (6) will be approximate. However, any errors in the initial conditions will be shown to have little effect on the modeling results.

From the foregoing discussion, it is clear that reconstructed $V_{r}, \beta_{r}, \alpha_{r}$ time series can have significant bias and drift errors, from the approximate initial conditions used in Eqs. (6), as well as the biases in the IMU data for angular rates $p, q, r$, and translational accelerations $a_{x}, a_{y}, a_{z}$. However, if the modeling is done in the frequency domain, where the bias and drift are removed for other reasons (discussed next), then this method of reconstructing air flow data can be used very effectively.

When transforming time-domain data into the frequency domain, the constant bias and drift (also called the trend, or the best-fit linear function of time) are always removed prior to applying the finite Fourier transform. This processing is done to avoid leakage from relatively large low-frequency components that can pollute the frequencydomain data at low frequencies of interest ${ }^{1,10,11}$. It follows that bias and drift errors in the air flow data reconstructions are of no concern when the analysis is done in the frequency domain, because the bias and drift of every time series are removed anyway. In essence, the reconstruction is done in the time domain, and the errors incurred in doing that are discarded prior to transformation into the frequency domain. At that point, standard frequency-domain modeling methods can be applied. Note that this approach conforms with the practical reality that angular rate measurements and translational accelerometer measurements typically have small but non-zero bias errors, and negligible slope or scale factor errors ${ }^{1}$.

To estimate the time skew in the angle of attack measurement, a simple nonlinear optimization can be done in the frequency domain. The model equation relating the measured angle of attack and the reconstructed angle of attack in the frequency domain is simply

$$
\tilde{\alpha}=\tilde{\alpha}_{r} e^{-j \omega \tau_{\alpha}}
$$

A nonlinear optimizer such as Gauss-Newton implemented in the frequency domain ${ }^{1}$ can be used to find the best estimate of the single unknown parameter $\tau_{\alpha}$. Equivalently, the parameter estimation problem can be set up as an output-error parameter estimation problem in the frequency domain, which is also solved using a nonlinear optimizer. This parameter estimation problem is very well conditioned and the solution is obtained very rapidly using Gauss-Newton optimization in the frequency domain. A similar approach can be used to find the time skews 
for airspeed and sideslip angle, individually. The angle of attack corrected for time skew in the frequency domain is computed by inverting Eq. (9) using the angle of attack time skew estimate $\hat{\tau}_{\alpha}$ found by the nonlinear optimizer,

$$
\tilde{\alpha}_{C}=\tilde{\alpha} e^{j \omega \hat{\tau}_{\alpha}}
$$

and similarly for airspeed and sideslip angle. Note that this approach retains data information from the air flow data measurements and only estimates relative time skews.

Once air flow angle data are corrected using the estimated time skews, the remaining aerodynamic modeling and control time skew estimation can be done in the frequency domain, as described in the next subsection.

Time skews in the Euler angle data from the IMU can be estimated in an analogous way using the nonlinear rotational kinematic equations of motion to reconstruct Euler angles $\phi, \theta, \psi$, from body-axis angular rates $p, q, r$. The rotational kinematic equations are ${ }^{1}$ :

$$
\begin{gathered}
\dot{\phi}_{r}=p+\tan \theta_{r}\left(q \sin \phi_{r}+r \cos \phi_{r}\right) \\
\dot{\theta}_{r}=q \cos \phi_{r}-r \sin \phi_{r} \\
\dot{\psi}_{r}=\frac{q \sin \phi_{r}+r \cos \phi_{r}}{\cos \theta_{r}}
\end{gathered}
$$

Reconstructed $\phi_{r}, \theta_{r}, \psi_{r}$ time series have no relative time skew, because the data reconstruction is based on IMU body-axis angular rate data. Equation (11c) can be omitted because Euler yaw angle does not affect dynamic modeling, and does not appear in Eqs. (11a)-(11b). Initial conditions for Eqs. (11a)-(11b) can be set to initial measured values or a mean of measured values in a steady initial flight condition. As mentioned previously, most IMU provide Euler angle time series that have been corrected for drift. However, filtering and data processing involved in producing the Euler angle time series can produce time skews relative to the angular rate data.

The time skew in the Euler pitch angle data can be estimated in the same manner as described earlier, using a simple nonlinear optimization in the frequency domain. For example, the model equation relating the Euler pitch angle data from the IMU and the reconstructed Euler pitch angle data in the frequency domain is simply

$$
\tilde{\theta}=\tilde{\theta}_{r} e^{-j \omega \tau_{\theta}}
$$

The Euler pitch angle data corrected for time skew in the frequency domain is computed by inverting Eq. (12) using the time skew estimate $\hat{\tau}_{\theta}$ found by the nonlinear optimizer,

$$
\tilde{\theta}_{c}=\tilde{\theta} e^{j \omega \hat{\tau}_{\theta}}
$$

The same approach can be applied for the Euler roll angle.

The rotational kinematic analysis can be done separately and independently from the translational kinematic analysis. Therefore, it is convenient to do the rotational analysis first, then correct the Euler angle data for the estimated time skews before doing the translational kinematic analysis to estimate the air flow data time skews. However, because the Euler angles appear only as arguments in trigonometric functions in the translational reconstruction Eqs. (6), and are not explanatory variables for the aerodynamic modeling, the rotational time skew estimation can be skipped.

\section{Aerodynamic Modeling and Control Time Skew Estimation in the Frequency Domain}

Continuing with the aircraft longitudinal short-period dynamics example described earlier, and using the angle of attack data corrected for time skew, the frequency-domain equations are: 


$$
\begin{gathered}
j \omega \tilde{\alpha}_{c}=Z_{\alpha} \tilde{\alpha}_{c}+\left(1+Z_{q}\right) \tilde{q}+Z_{\delta_{e}} \tilde{\delta}_{e} e^{-j \omega \tau_{\delta_{e}}} \\
j \omega \tilde{q}=M_{\alpha} \tilde{\alpha}_{c}+M_{q} \tilde{q}+M_{\delta_{e}} \tilde{\delta}_{e} e^{-j \omega \tau_{\delta_{e}}} \\
\tilde{a}_{z}=\frac{V_{o}}{g}\left[Z_{\alpha} \tilde{\alpha}_{c}+Z_{q} q+Z_{\delta_{e}} \tilde{\delta}_{e} e^{-j \omega \tau_{\delta_{e}}}\right]
\end{gathered}
$$

As mentioned earlier, the remaining unknown parameters, consisting of stability and control derivatives and control time skew parameter, can now be estimated using standard parameter estimation methods in the frequency domain.

Using an equation-error formulation, Eqs. (14) can be analyzed either individually or together. The equationerror approach produces parameter estimates that minimize the least squares fit of the model to derivative information in the frequency domain for each equation ${ }^{1}$. For the pitching moment equation (14b), the equation error in the frequency domain is

$$
\tilde{\varepsilon}(\boldsymbol{\theta})=\tilde{z}-\tilde{y}(\boldsymbol{\theta})
$$

where

$$
\begin{gathered}
\tilde{z}=j \omega \tilde{q} \\
\tilde{y}(\boldsymbol{\theta})=M_{\alpha} \tilde{\alpha}_{c}+M_{q} \tilde{q}+M_{\delta_{e}} \tilde{\delta}_{e} e^{-j \omega \tau_{\delta_{e}}} \\
\boldsymbol{\theta}=\left[\begin{array}{llll}
M_{\alpha} & M_{q} & M_{\delta_{e}} & \tau_{\delta_{e}}
\end{array}\right]^{T}
\end{gathered}
$$

The Fourier transform quantities based on the data, i.e., $\tilde{\alpha}_{c}, \tilde{q}, \tilde{\delta}_{e}$, are computed at $M$ selected frequencies $\omega_{k}, k=1,2, \ldots, M$. The number of frequencies $M$ is selected to be much greater than the number of unknown parameters $n_{p}$ ( $n_{p}=4$ in Eq. (16c)), and the frequencies are chosen to cover the frequency band associated with the dynamic model, e.g., $[0.1,1.5] \mathrm{Hz}$ for rigid-body dynamics of a typical fighter or subscale aircraft. Note that the transform frequencies can be chosen arbitrarily (subject only to data information content limitations) using the highaccuracy numerical method described in Refs. [1] and [9]. This results in an overdetermined set of equations with complex data,

$$
\tilde{\mathbf{z}}=\tilde{\boldsymbol{y}}(\boldsymbol{\theta})+\tilde{\boldsymbol{\varepsilon}}
$$

where

$$
\begin{aligned}
& \tilde{\mathbf{z}}=\left[\begin{array}{lllll}
j \omega_{1} \tilde{q}\left(\omega_{1}\right) & j \omega_{2} \tilde{q}\left(\omega_{2}\right) & \ldots & j \omega_{M} & \tilde{q}\left(\omega_{M}\right)
\end{array}\right]^{T} \\
& \tilde{\boldsymbol{\alpha}}_{c}=\left[\begin{array}{llll}
\tilde{\alpha}_{c}\left(\omega_{1}\right) & \tilde{\alpha}_{c}\left(\omega_{2}\right) & \ldots & \tilde{\alpha}_{c}\left(\omega_{M}\right)
\end{array}\right]^{T} \\
& \tilde{\boldsymbol{q}}=\left[\begin{array}{llll}
\tilde{q}\left(\omega_{1}\right) & \tilde{q}\left(\omega_{2}\right) & \ldots & \tilde{q}\left(\omega_{M}\right)
\end{array}\right]^{T} \\
& \boldsymbol{\delta}_{e}=\left[\begin{array}{llll}
\tilde{\delta}_{e}\left(\omega_{1}\right) & \tilde{\delta}_{e}\left(\omega_{2}\right) & \ldots & \tilde{\delta}_{e}\left(\omega_{M}\right)
\end{array}\right]^{T}
\end{aligned}
$$




$$
\begin{gathered}
\boldsymbol{\omega}=\left[\begin{array}{llll}
\omega_{1} & \omega_{2} & \ldots & \omega_{M}
\end{array}\right]^{T} \\
\tilde{\boldsymbol{y}}(\boldsymbol{\theta})=M_{\alpha} \tilde{\boldsymbol{\alpha}}_{C}+M_{q} \tilde{\boldsymbol{q}}+M_{\delta_{e}} \tilde{\boldsymbol{\delta}}_{e} e^{-j \boldsymbol{\omega} \tau_{\delta_{e}}}
\end{gathered}
$$

and $\tilde{\boldsymbol{\varepsilon}}$ is a vector of complex equation errors. Equation (18f) shows that the model output is nonlinearly dependent on the parameters, because of the time skew parameter $\tau_{\delta_{e}}$ in the exponential. This simply means that the solution cannot be obtained with the algebraic pseudo-inverse calculation normally used for equation-error parameter estimation, but rather must be solved using a nonlinear optimizer, such as Gauss-Newton in the frequency domain. The solution is obtained in a straightforward manner using nonlinear optimization (typically applied for other nonlinear parameter estimation, such as output-error problems) in the frequency domain, with the least squares cost function:

$$
J(\theta)=\frac{1}{2}[\tilde{z}-\tilde{y}(\theta)]^{\dagger}[\tilde{z}-\tilde{y}(\theta)]
$$

The process produces accurate estimates for the stability and control parameters, as well as the control time skew. For these estimated parameters, as well as the air flow time skew parameters (which also come from a nonlinear parameter estimation solution), the estimated parameter covariance matrix can be computed from ${ }^{1}$

$$
\begin{gathered}
\boldsymbol{\Sigma}(\hat{\boldsymbol{\theta}}) \equiv E\left[(\hat{\boldsymbol{\theta}}-\boldsymbol{\theta})(\hat{\boldsymbol{\theta}}-\boldsymbol{\theta})^{T}\right]=\hat{\sigma}^{2}\left\{\operatorname{Re}\left[\boldsymbol{S}^{\dagger} \boldsymbol{S}\right]\right\}^{-1} \\
\left.\boldsymbol{S} \equiv \frac{\partial \tilde{\boldsymbol{y}}(\boldsymbol{\theta})}{\partial \boldsymbol{\theta}}\right|_{\boldsymbol{\theta}=\hat{\boldsymbol{\theta}}}
\end{gathered}
$$

where the equation-error variance estimate $\hat{\sigma}^{2}$ can be found from the model residuals,

$$
\hat{\sigma}^{2}=\frac{1}{\left(M-n_{p}\right)}\left\{[\tilde{\boldsymbol{z}}-\tilde{\boldsymbol{y}}(\hat{\boldsymbol{\theta}})]^{\dagger}[\tilde{\boldsymbol{z}}-\tilde{\boldsymbol{y}}(\hat{\boldsymbol{\theta}})]\right\}
$$

and $n_{p}$ is the number of unknown parameters, i.e., the number of elements in parameter vector $\boldsymbol{\theta}$. The standard errors of the estimated parameters are given by the square roots of the diagonal elements of the covariance matrix $\Sigma(\hat{\boldsymbol{\theta}})$,

$$
s\left(\hat{\theta}_{j}\right)=\sqrt{\operatorname{Var}\left(\hat{\theta}_{j}\right)}=\sqrt{\Sigma_{j j}} \quad j=1,2, \ldots, n_{p}
$$

Explanations of why the estimated parameter standard errors are computed in this way, and why this calculation in the frequency domain produces parameter error measures that are consistent with the scatter in parameter estimates from repeated maneuvers, can be found in Ref. [1].

It is also possible to solve this parameter estimation problem using an output-error formulation in the frequency domain, which is equivalent to finding model parameter estimates that match the aircraft response information in a least squares sense, rather than the derivative information. However, that approach is more complicated, and it was found that the equation-error formulation provided better parameter sensitivity for the control time skew parameters, and produced modeling results with high accuracy. Because the nonlinear optimization is being done in the frequency domain, all of the calculations are algebraic, and the nonlinear optimization can be carried out very rapidly on a desktop computer. 
Using the equation-error approach described here, each equation (for example, Eq. (14b) or (14c)) can be analyzed individually. However, model parameters can also be estimated in a combined equation-error formulation. For example, Eqs. (14b) and (14c) could be combined, and the nonlinear optimization applied to the combined frequency-domain data. This allows the estimator to find a single best value for the control time skew, which appears in both Eqs. (14b) and (14c).

Experience has shown that using the output equation (14c) for $a_{z}$ gives superior modeling results compared to using the state equation (14a) for $\alpha$, when estimating the model parameters that appear in both equations. This is related to improved parameter sensitivity and signal-to-noise ratio using the output equation (14c). Similar statements apply for lateral/directional modeling, relative to using the output equation for $a_{y}$ versus the state equation for $\beta$.

Although the example shown here is for longitudinal short-period dynamics using dimensional stability and control derivatives, the same approach can be used for other models, including lateral/directional dynamic models. Furthermore, the approach also applies if the modeling is done using nondimensional aerodynamic coefficients and nondimensional stability and control derivatives. In that case, the measured data to be matched by the model using the equation-error formulation are nondimensional aerodynamic force and moment coefficient data, computed from flight measurements as follows ${ }^{1}$ :

$$
\begin{gathered}
C_{X}=\left(m a_{x}-T_{x}\right) / \bar{q} S \\
C_{Z}=\left(m a_{z}-T_{z}\right) / \bar{q} S \\
C_{m}=\left[I_{y} \dot{q}+\left(I_{x}-I_{z}\right) p r+I_{x z}\left(p^{2}-r^{2}\right)-M_{T}\right] / \bar{q} S \bar{c} \\
C_{Y}=m a_{y} / \bar{q} S \\
C_{l}=\left[I_{x} \dot{p}-I_{X z}(p q+\dot{r})+\left(I_{z}-I_{y}\right) q r\right] / \bar{q} S b \\
C_{n}=\left[I_{z} \dot{r}-I_{x z}(\dot{p}-q r)+\left(I_{y}-I_{x}\right) p q\right] / \bar{q} S b
\end{gathered}
$$

These expressions retain the full nonlinear dynamics in the equations of motion for a symmetric rigid aircraft. For local modeling, the aerodynamic force and moment coefficients computed from Eqs. (21) and (22) can be modeled using linear expansions in aircraft states and controls, e.g.,

$$
\begin{gathered}
C_{X}=C_{X_{\alpha}} \alpha+C_{X_{q}} \frac{q \bar{c}}{2 V}+C_{X_{\delta}} \delta \\
C_{Z}=C_{Z_{\alpha}} \alpha+C_{Z_{q}} \frac{q \bar{c}}{2 V}+C_{Z_{\delta}} \delta \\
C_{m}=C_{m_{\alpha}} \alpha+C_{m_{q}} \frac{q \bar{c}}{2 V}+C_{m_{\delta}} \delta \\
C_{Y}=C_{Y_{\beta}} \beta+C_{Y_{p}} \frac{p b}{2 V}+C_{Y_{r}} \frac{r b}{2 V}+C_{Y_{\delta}} \delta \\
C_{l}=C_{l_{\beta}} \beta+C_{l_{p}} \frac{p b}{2 V}+C_{l_{r}} \frac{r b}{2 V}+C_{l_{\delta}} \delta
\end{gathered}
$$




$$
C_{n}=C_{n_{\beta}} \beta+C_{n_{p}} \frac{p b}{2 V}+C_{n_{r}} \frac{r b}{2 V}+C_{n_{\delta}} \delta
$$

where all aircraft states and controls are perturbations from a reference condition. In each expansion, a single term is shown to represent all relevant and similar control terms, to simplify the expressions. For example, in Eq. (24b), the term $C_{l_{\delta}} \delta$ represents all the control terms for $C_{l}$, e.g., $C_{l_{\delta}} \delta \equiv C_{l_{\delta_{a}}} \delta_{a}+C_{l_{\delta_{r}}} \delta_{r}$. For a typical stability and control flight test maneuver, dynamic pressure $\bar{q}$ is relatively constant or changes slowly, so that small time skews in the $\bar{q}$ data will have little effect on the modeling results. Similar statements apply for the thrust terms.

At this stage, the time skew parameters are introduced, the time series are transformed into the frequency domain, and the modeling proceeds in the same way as described earlier. Note that the linear expansions in Eqs. (23)-(24) do not include bias terms, because modeling in the frequency domain excludes these terms, as a result of detrending the time series prior to transforming the time series into the frequency domain, which is equivalent to projecting the time-domain data onto complex sinusoidal basis functions with selected non-zero frequencies. If the bias and linear drift with time are of interest, they can be estimated using a follow-on estimation in the time domain, which is a well-conditioned and simple problem to solve ${ }^{12}$.

The linear aerodynamic models in Eqs. (23) and (24) contain parameters called nondimensional stability and control derivatives, such as $C_{l_{\beta}}$ and $C_{m_{\delta_{e}}}$, which characterize the stability and control of the aircraft.

\section{Test Aircraft}

\section{A. F-16 Nonlinear Simulation}

The F-16 is a single-seat, multi-role fighter with a blended wing / body and a cropped delta wing planform with leading edge sweep of $40 \mathrm{deg}$. Thrust is provided by one General Electric F110-GE-100 or Pratt \& Whitney F100-PW-220 afterburning turbofan engine mounted in the rear fuselage. Figure 2 shows a photograph of the F-16. Mass properties and geometry of the aircraft are given in Table 1 .

The aircraft was modeled with controls for throttle $\delta_{t h}$, stabilator $\delta_{s}$, aileron $\delta_{a}$, and rudder $\delta_{r}$. Speed brake and flaps were assumed fixed at zero deflection. Throttle deflection was limited to the range $0 \leq \delta_{\text {th }} \leq 1$, stabilator deflection was limited to $-25^{\circ} \leq \delta_{s} \leq 25^{\circ}$, aileron deflection was limited to $-21.5^{\circ} \leq \delta_{a} \leq 21.5^{\circ}$, and rudder deflection was

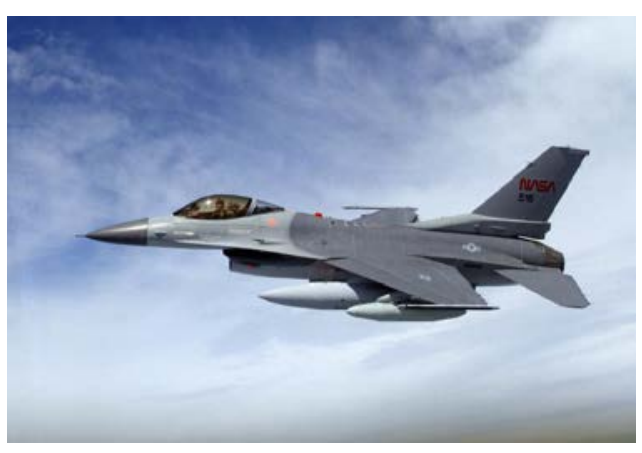

Figure 2. F-16 Aircraft

Credit: NASA Langley Research Center limited to $-30^{\circ} \leq \delta_{r} \leq 30^{\circ}$. These limits represent the physicals stops.

Nondimensional aerodynamic force and moment coefficient data were derived from a low-speed static wind tunnel test and a dynamic forced oscillation wind tunnel test, both conducted with a 16\% scale model of the F- 16 . The aerodynamic database applies to the F-16 flown out of ground effect, with landing gear retracted, and no external stores. Static aerodynamic data were in tabular form as a function of angle of attack and sideslip angle over the ranges $-10^{\circ} \leq \alpha \leq 45^{\circ}$ and $-30^{\circ} \leq \beta \leq 30^{\circ}$, respectively. Dynamic data were provided in tabular form at zero sideslip angle over the angle of attack range $-10^{\circ} \leq \alpha \leq 45^{\circ}$. Dependence of the nondimensional coefficients on $\dot{\alpha}$ was included in the $q$ dependencies, due to the manner in which the data were collected in the wind tunnel.

The engine model was based on ground test data in tabular form, with thrust given as a function of altitude, Mach number, and engine power level. Engine power level was computed from throttle position and throttle gearing, including first order dynamics for the dynamic thrust response.

The F-16 nonlinear simulation was programmed completely in MATLAB ${ }^{\circledR}$. Full nonlinear equations of motion, including turbine engine gyroscopic effects, were used. Complete details on the F-16 nonlinear simulation can be found in Appendix D of Ref. [1]. 


\section{B. T-2 Subscale Jet Transport Aircraft}

The T-2 aircraft is a 5.5 percent dynamically-scaled model of a generic commercial twin-engine jet transport aircraft. A photograph of the aircraft in flight is shown in Fig. 3. The aircraft has twin jet engines mounted under the wings and retractable tricycle landing gear. Aircraft geometry and nominal mass properties are given in Table 1. Further information on the T-2 subscale jet transport aircraft and associated flight test operations can be found in Refs. [13]-[16].

\section{Control Surfaces}

Control surfaces on the T-2 aircraft are left and right ailerons, left and right inboard and outboard elevators, upper and lower rudders, left and right inboard and

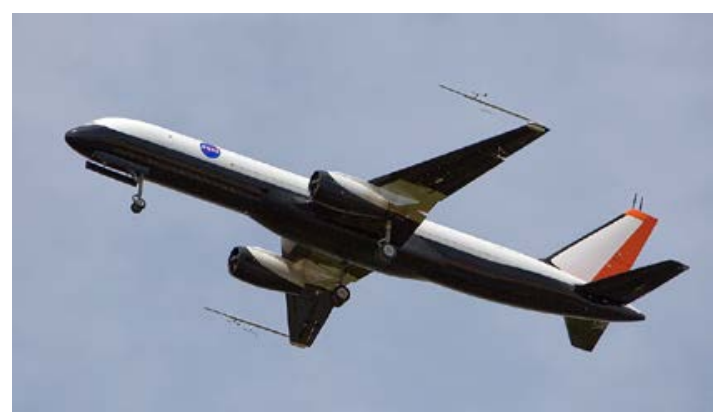

Figure 3. T-2 subscale jet transport aircraft

Credit: NASA Langley Research Center outboard trailing-edge flaps, and left and right inboard and outboard spoilers, for a total of 16 independent control surfaces. For the flight data analyzed in this work, only the elevators, ailerons, and rudders were deflected. The individual elevator surfaces were moved together as a single elevator surface, and similarly for the rudders. Left and right ailerons were deflected asymmetrically, in the conventional way. Definitions of control surface deflections are given below. Trailing edge down is positive deflection for wing and elevator surfaces, and trailing edge left is positive for rudder surfaces.

$$
\begin{gathered}
\delta_{e}=\frac{1}{4}\left(\delta_{e_{L O}}+\delta_{e_{L I}}+\delta_{e_{R I}}+\delta_{e_{R O}}\right) \\
\delta_{a}=\frac{1}{2}\left(\delta_{a_{R}}-\delta_{a_{L}}\right) \quad \delta_{r}=\frac{1}{2}\left(\delta_{r_{\text {upper }}}+\delta_{r_{\text {lower }}}\right)
\end{gathered}
$$

A research pilot executed the flight test maneuvers from inside a mobile control room, using a synthetic vision display drawn from telemetry data and a local terrain database, along with video from a camera in the nose of the aircraft. Inputs from the research pilot and a ground-based flight control system were used to generate control surface commands which were transmitted by telemetry to the aircraft. The aircraft can also be flown by a safety pilot using direct visual contact and conventional radio control.

The flight control system has the capability to inject automated control surface perturbations to excite the aircraft dynamic response for modeling purposes. These control surface perturbations can have arbitrary waveforms, and can be applied to multiple control surfaces individually or simultaneously. The perturbations are summed with pilot and feedback control commands in the flight control system, just before the actuator command rate and position limiting. Typically, the research pilot flies the aircraft to the desired flight condition(s), then initiates the automated control surface perturbations by holding in a button switch on the throttle.

\section{Instrumentation and Data Acquisition}

The T-2 aircraft was equipped with a micro-INS, which provided 3-axis translational accelerometer measurements, angular rate measurements, estimated attitude angles, and GPS velocity and position. Air data probes attached to booms mounted on each wingtip (visible in Fig. 3) measured angle of attack, sideslip angle, static pressure, and dynamic pressure. Measurements from static pressure sensors and ambient temperature sensors were used to compute air density and altitude. Engine speeds in rpm were measured and used as inputs to an engine model to compute thrust. The engine model was identified from ground test data, with adjustments for ram drag identified from flight data. Potentiometers on the rotation axes of the control surfaces measured control surface deflections. Mass properties were computed based on measured fuel flow, pre-flight weight and balance, and inertia measurements done on the ground for the aircraft without fuel. Pilot stick and rudder pedal commands and throttle position were also measured and recorded. Data from onboard sensors were telemetered to the ground in real time. Sampling rate for the flight data was $200 \mathrm{~Hz}$, downsampled to $50 \mathrm{~Hz}$ for data analysis and dynamic modeling. 


\section{Results}

\section{A. Simulation Results}

The method for dynamic modeling based on flight data with unknown relative time skews was first applied to data from the F-16 nonlinear simulation. Orthogonal optimized multisine excitations ${ }^{1}$ were applied simultaneously to the stabilator, ailerons, and rudder. Gaussian random noise with magnitude for approximately a 20:1 signal-tonoise ratio (five percent noise, based on simulation output root-mean-square values) was added to all simulation outputs, and typical bias errors $(0.1 \mathrm{deg} / \mathrm{s}$ for the angular rate sensors and $0.01 \mathrm{~g}$ for the translational accelerometers) were applied. Time skew equal to $0.10 \mathrm{~s}$ (time delay) was applied to airspeed, sideslip angle, and angle of attack data, and time skew equal to $-0.10 \mathrm{~s}$ (time advance) was applied to the controls. These time skew values were chosen as arbitrary but realistic values, for demonstration purposes. Figure 4 shows simulated F-16 longitudinal flight data without time skews ("time synch") and with time skews ("time skew"). Figure 5 shows airspeed, sideslip angle, and angle of attack data from the maneuver, compared to the data reconstruction using other measurements. Inaccurate initial conditions for the data reconstruction were purposely introduced. The time-domain reconstruction clearly shows bias and drift errors, as well as time skews in the air flow data.

The corrected time skew method described in Section III was applied to the time skewed data, with parameter estimation results shown in column 2 of Table 2, with the heading Corrected Time Skew Method. Equation-error in the frequency domain was formulated using both the pitching moment equation (2b) and the body-axis $z$ acceleration output equation (2c) simultaneously, with a single unknown time skew parameter for the stabilator. The transformation of time domain data into the frequency domain was done using the frequencies:

$$
f=\left[\begin{array}{lllll}
2 / T & 2 / T+0.02 & 2 / T+0.04 & \ldots & 2.0
\end{array}\right] \mathrm{Hz}
$$

where $T$ was the time length of the maneuver in seconds. This selection of frequencies includes at least two cycles for each frequency during the length of the maneuver, with a very fine frequency resolution to capture details in the frequency domain. The $2 \mathrm{~Hz}$ upper limit for the frequency band was selected so that all deterministic frequency content was included, but wideband noise was automatically filtered by the transformation. This approach enhances signal-to-noise ratio, and improves modeling results, while retaining all useful information for parameter estimation in the frequency domain. Conversion to the frequency domain was done using the high-accuracy finite Fourier transform ${ }^{1,9}$ mentioned earlier, which allows selection of the transformation frequencies, independent of the length of the maneuver $T$. The accuracy of this numerical calculation is on the order of the round-off error of the computer.

Equation-error parameter estimation in the frequency domain was then applied to simulated noisy data without time skews, with the results shown in column 3 of Table 2. Comparing results in columns 2 and 3 of Table 2 shows that the corrected time skew method produced model parameter estimates from time-skewed data with roughly the same accuracy and uncertainty as equation-error in the frequency domain applied to data without time skews. This demonstrates that the proposed method successfully solves the problem it was designed to solve. Previous realistic simulation testing and flight test data analysis have shown that the accuracy of model parameters estimated using equation-error in the frequency domain is comparable to using a time-domain output-error method employing iterative nonlinear optimization ${ }^{1,10,17}$. The proposed method also estimated the time skews in the data very accurately, as shown in the last two rows of column 2 in Table 2. Column 4 of Table 2 shows the inaccurate parameter estimation results obtained from time-skewed data using equation-error in the frequency domain. These results highlight the need for the proposed method when the data are time skewed.

Figure 6 is a graphical representation of the parameter estimation results given in Table 2. The vertical bars shown in Fig. 6 indicate the 95 percent confidence intervals, or $\hat{\theta} \pm 2 \hat{\sigma}$. The parameters most adversely affected by time skews in the data were the $M_{\alpha}$ and $M_{q}$ stability derivatives. 

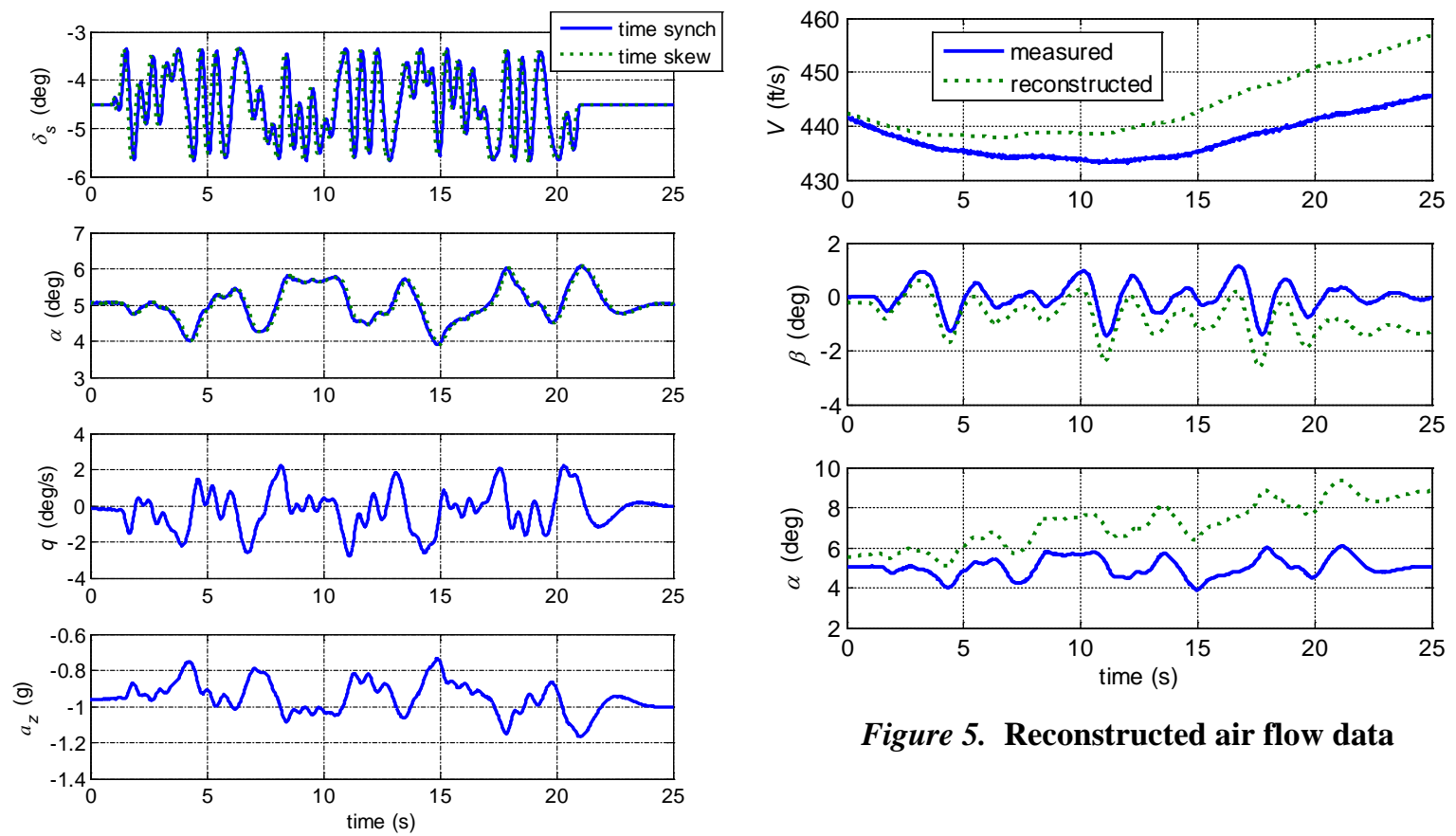

Figure 5. Reconstructed air flow data

Figure 4. Simulated F-16 flight data
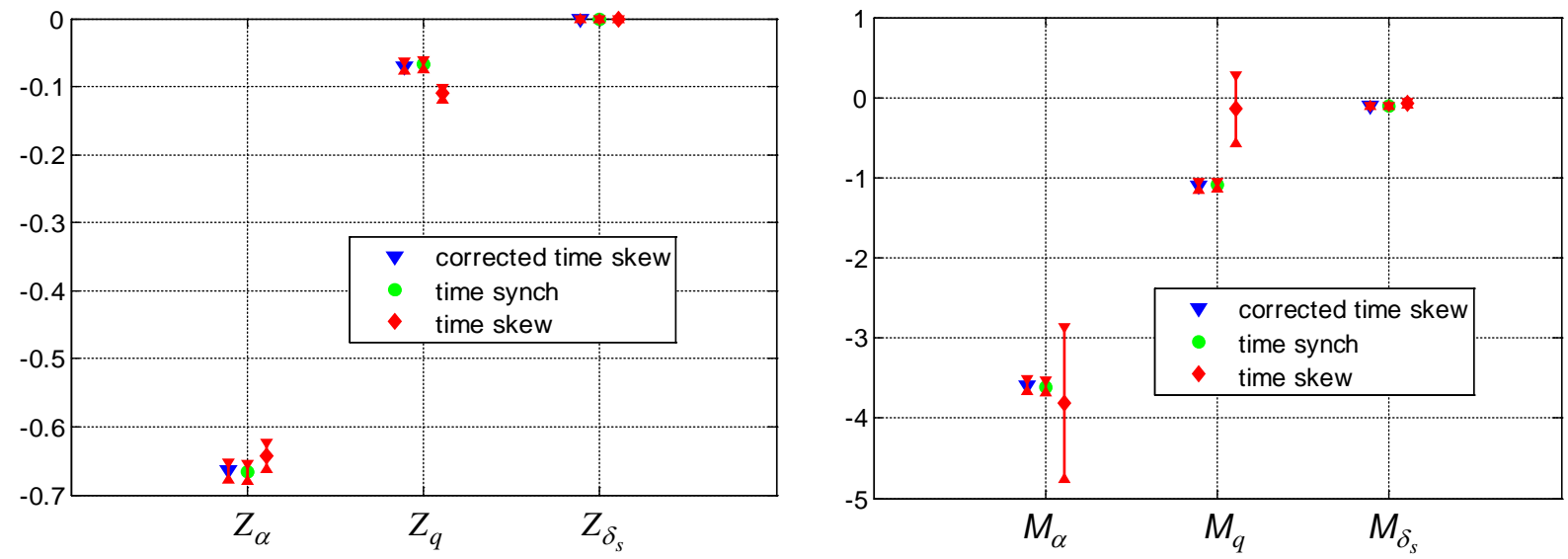

Figure 6. F-16 longitudinal parameter estimation results

A Monte Carlo analysis was done by selecting angular rate and translational acceleration biases, as well as air flow and control time skews, from specified intervals using a uniform probability density, and applying varying white Gaussian noise sequences to the simulation outputs. The angular rate biases were chosen on the interval $[-0.5,0.5] \mathrm{deg} / \mathrm{s}$ and translational acceleration biases were chosen on the interval $[-0.02,0.02] \mathrm{g}$. Air flow and control time skews were chosen individually from the interval $[-0.20,0.20] \mathrm{s}$. Parameter estimation results were computed using the method described in Section III, and using equation-error in the frequency domain applied to data without time skews. In addition, parameter estimation results were computed by applying equation-error in the frequency domain to time-skewed data, to demonstrate the detrimental effects of time-skewed data on parameter 
estimation results. The entire analysis was repeated 200 times with the same input sequences applied to the control surfaces, but with different instrumentation biases, time skews, and random noise sequences for each run.

Table 3 shows parameter estimation results from the Monte Carlo runs. The standard errors were computed from the scatter in the parameter estimates,

$$
\begin{gathered}
\hat{\sigma}_{\theta}=\sqrt{\frac{1}{(n-1)} \sum_{i=1}^{n}\left(\hat{\boldsymbol{\theta}}_{i}-\overline{\boldsymbol{\theta}}\right)^{2}} \\
\overline{\boldsymbol{\theta}}=\frac{1}{n} \sum_{i=1}^{n} \hat{\boldsymbol{\theta}}_{i}
\end{gathered}
$$

where $n$ is the total number of Monte Carlo runs. The computed $\hat{\sigma}_{\theta}$ quantifies the scatter in repeated parameter estimates. Figure 7 shows the results graphically, using 95 percent confidence intervals, as in Fig. 6.

The Monte Carlo results show that the proposed modeling method for time-skewed data (Table 3, column 2, and "corrected time skew" in Fig. 7) is accurate in terms of the mean value of the parameter estimates, but exhibits slightly larger scatter in the individual estimates from each run, compared to using equation-error in the frequency domain and data without time skews (Table 3, column 3, and "time synch" in Fig. 7). However, in both cases, the scatter for repeated estimates was small. In addition, time skew parameters were estimated very accurately using the proposed method, with root-mean-square errors less than $0.018 \mathrm{~s}$. The parameter estimation results obtained using equation-error in the frequency domain with time-skewed data (Table 3, column 4, and "time skew" in Fig. 7) show biased parameter estimates with large scatter, which highlights the need for the proposed method.

Error bounds for the parameter estimates can be lowered by running maneuvers repeatedly and averaging the individual parameter estimates, or analyzing the data from repeated maneuvers together. Because the proposed method is accurate in the mean, improved parameter accuracy can be obtained with time-skewed data using repeated maneuvers to compensate for the slightly larger scatter in the parameter estimates.

Parameter estimation results from the Monte Carlo analysis demonstrate that accurate modeling results can be obtained by applying the proposed method to noisy time-skewed data with realistic bias errors in the angular rate and translational acceleration measurements.
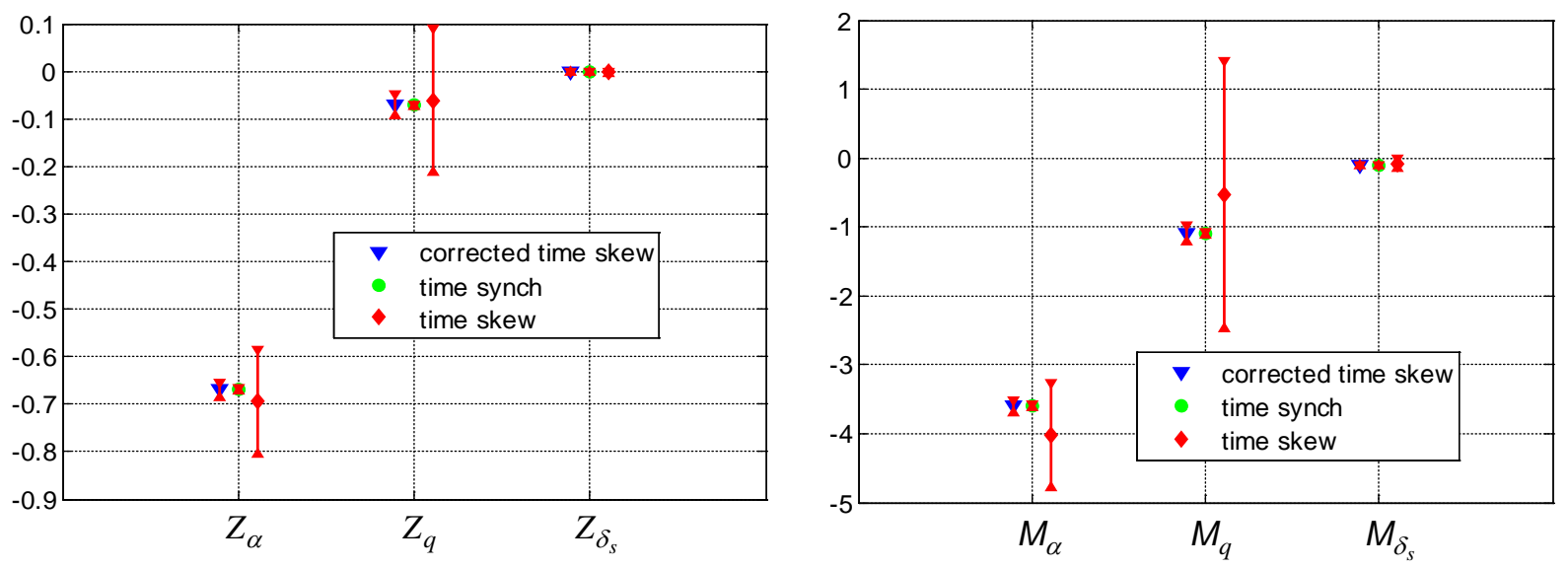

Figure 7. F-16 longitudinal parameter estimation results for 200 Monte Carlo runs 


\section{B. Flight Test Results}

After the modeling method was successfully developed and validated using F-16 nonlinear simulation data, the same approach was applied to flight data from the T-2 subscale jet transport aircraft. This section contains the flight results and associated discussion.

Figure 8 shows flight data from the T-2 aircraft during a maneuver where orthogonal optimized multisine inputs ${ }^{1}$ were applied simultaneously to the elevator, aileron, and rudder controls. The nominal flight condition was 4.6 deg angle of attack and $130 \mathrm{ft} / \mathrm{s}$ airspeed, at $750 \mathrm{ft}$ altitude. Measured flight data included both control surface commands and control surface positions. The elevator command and position data are shown in the top plot of Fig. 8. The elevator position time series is roughly a lagged version of the elevator command time series, so that a simple time lag can be used as an approximate model for the actuator and control linkage. This provided an opportunity to test the proposed method using control data that is known to be time skewed.

Using an approach similar to the F-16 nonlinear simulation testing, parameter estimation results were computed by applying the method described in Section III to the flight data, using control command data as the input. Equation-error in the frequency domain was applied to flight data that included control position data as the input, along with angle of attack data corrected for the time skew estimated by the frequency-domain data reconstruction technique described in Section III. In addition, parameter estimation results were computed by applying equationerror in the frequency domain to time-skewed flight data, which included control command data as the input and uncorrected angle of attack data.

Table 4 shows the flight results in a format similar to Table 2. Figure 9 shows the model fit to the data in the frequency domain using the proposed method, and Fig. 10 shows the corresponding model fit to the data in the time
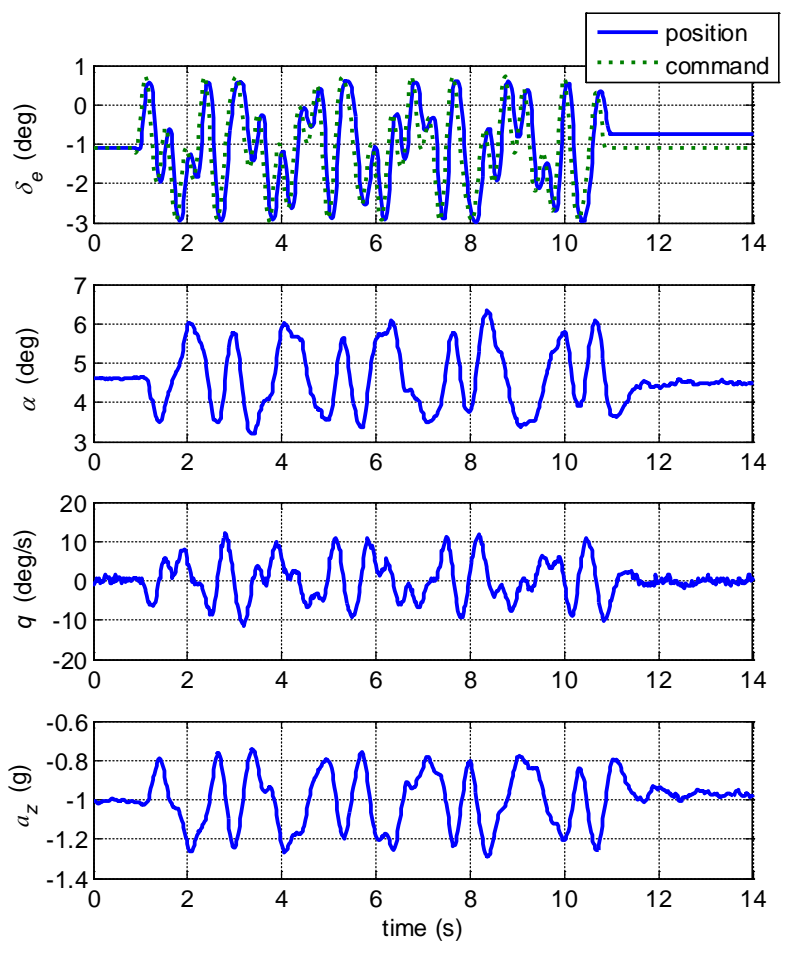

Figure 8. T-2 flight data domain. Both of these model fits are degraded slightly by the fact that a pure time shift is only an approximate model for the elevator actuator and control linkage, and the measured angle of attack includes the effects of imperfections in the air flow angle measurements from the wingtip vanes, such as the structural response of the wings and wingtip booms during dynamic maneuvering, turbulence, inertia and friction in the vane mechanisms, and local flow effects, typically called upwash. These effects do not appear in the reconstructed angle of attack data. Nevertheless, the model fits in both the time and frequency domains are excellent. Figure 11 is a graphical depiction of the modeling results in Table 4, presented in the same way as in Fig. 6.

As in the F-16 simulation case, the parameter estimates computed by applying the proposed method to time-skewed data showed good consistency but slightly larger uncertainties, compared to the parameter estimates computed using time-synchronized data. The modeling results in this case demonstrate some robustness to the practical effects listed earlier, which were not modeled in the F-16 nonlinear simulation. As in the simulation case, applying frequency-domain equation error parameter estimation to time-skewed data produced inaccurate parameter estimates with large error bounds.

The estimated time skew in the angle of attack data relative to the IMU data matched well with a separate estimate in the time domain based on the least squares difference between detrended measured angle of attack data and detrended reconstructed angle of attack data, see Fig. 12. The angle of attack time skew estimated in this way matched the frequency domain estimate to within the error bound of the frequency-domain estimate. Note that the measured and reconstructed angle of attack data in the lower plot of Fig. 12 have been detrended, to highlight the 
time skew. Without the detrending, this plot would look more like the lower plot in Fig. 5. Figure 13 is a magnified view of the upper plot in Fig. 12, showing that the control time skew estimate found by the method described in Section III (-0.0750 s from Table 4) was an excellent match to the known time skew shown in Fig. 13.

\section{Conclusions}

A method for accurately estimating dynamic model parameters from flight data with unknown time skews was developed and demonstrated using data from an F-16 nonlinear simulation and flight data from a subscale aircraft. The technique combines data reconstruction, nonlinear optimization, and equation-error parameter estimation in the frequency domain to create a simple and accurate method for dynamic modeling based on time-skewed data. The approach can also be used to accurately quantify time skews in the data, and therefore can be useful as a diagnostic tool. Although the technique was demonstrated using a longitudinal dynamic model with dimensional aerodynamic derivatives, the approach could be used just as well for nondimensional aerodynamic derivatives, and/or for lateral/directional dynamic models.

The technique developed for estimating air flow data time skews can be used for any type of maneuvering, not just the small perturbation maneuvers shown in the examples. For control time skew estimation, the aerodynamic model must be linear in the controls, but could be nonlinear otherwise.

An application of this approach is subscale aircraft flight testing, where control surface command data are used rather than direct measurements of control position. Assuming the actuator dynamics can be approximated by a simple time lag, this modeling approach can be used to estimate aerodynamic parameters using only control surface command data, or data that may be time skewed because of telemetry delays, low-bandwidth actuators, or control linkages under flight loads. The approach can also be useful when air flow angle data come from pressure probes or flush air data systems, which may have pneumatic time lags. The technique could also be applied to accident data or operational flight data, which often have unknown time skews, and could be useful as a diagnostic tool for relative time skews in flight data.
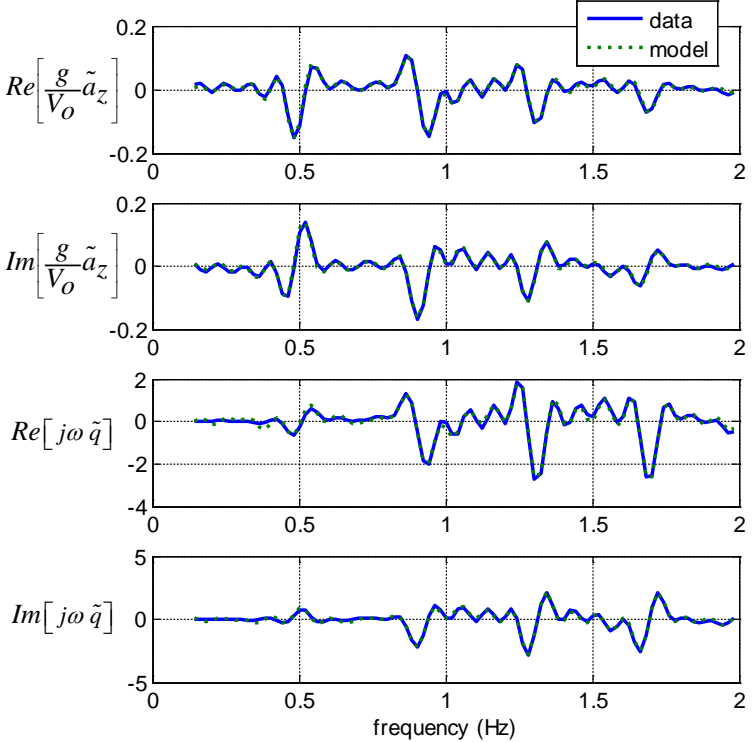

Figure 9. T-2 frequency-domain model fit
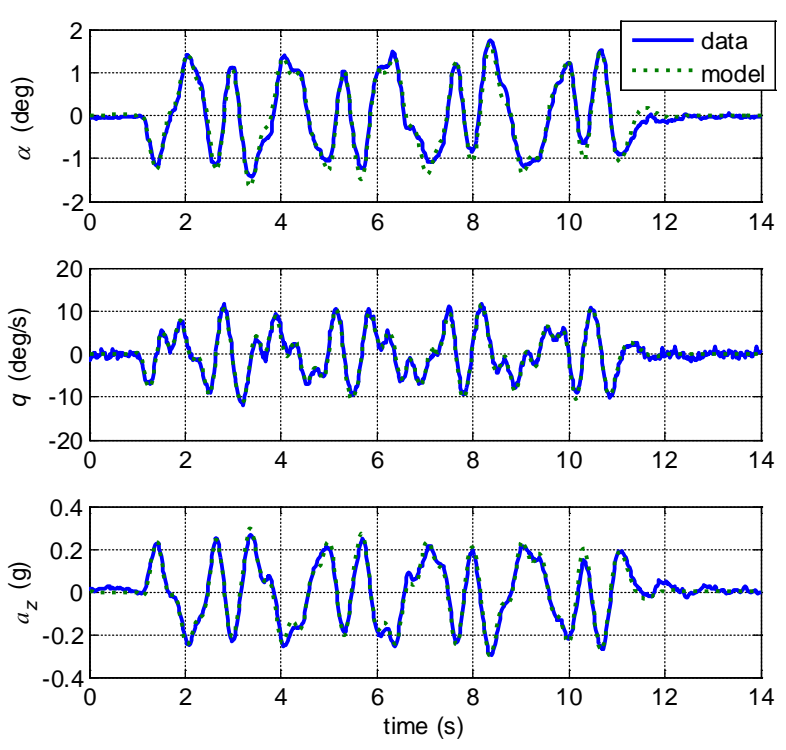

Figure 10. T-2 time-domain model fit 

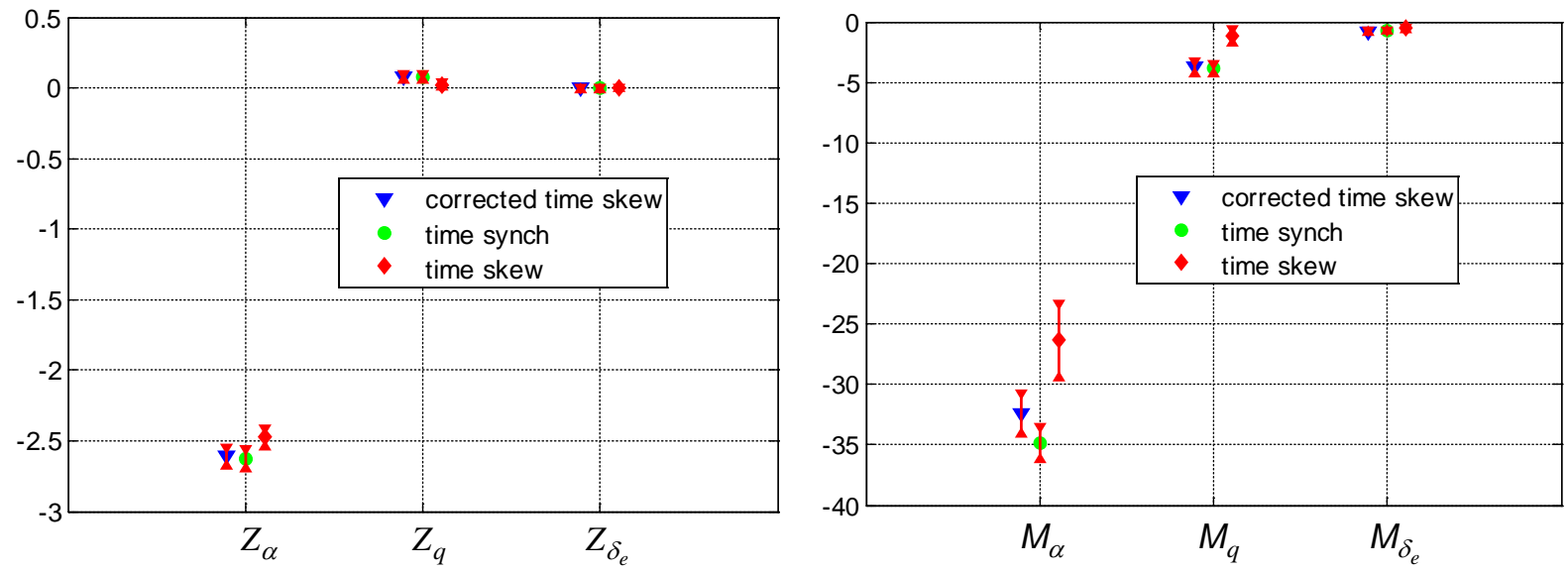

Figure 11. T-2 longitudinal parameter estimation results
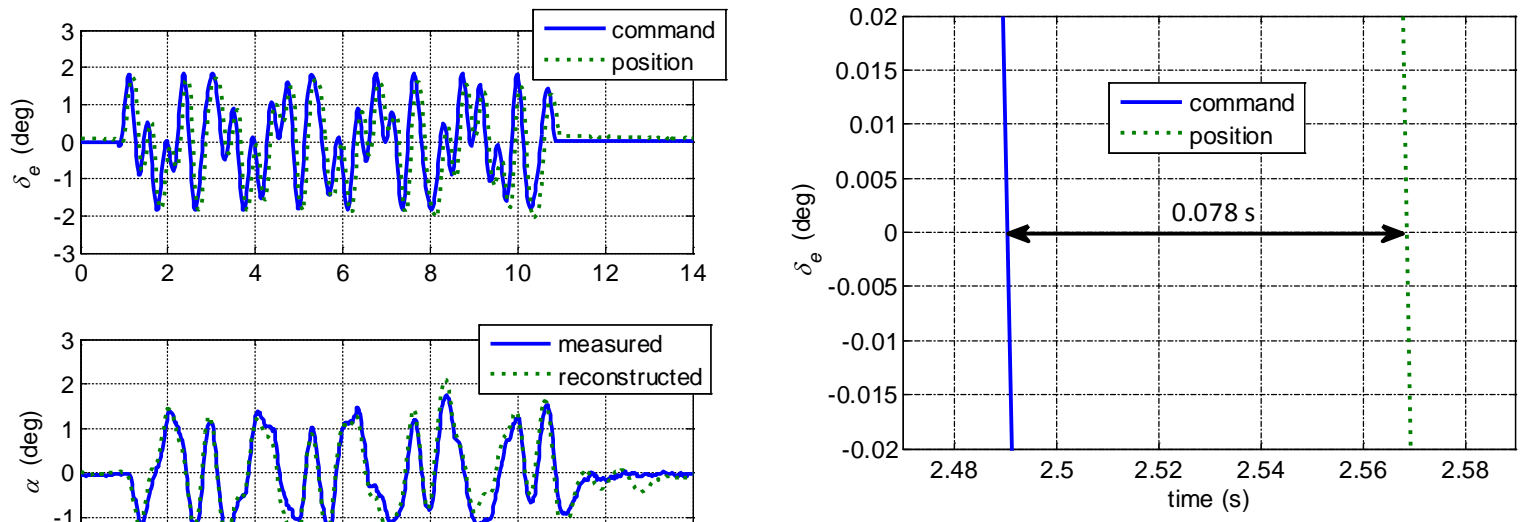

Figure 13. Detail view of T-2 detrended control data

Figure 12. T-2 detrended data

\section{Acknowledgments}

The efforts of the AirSTAR flight test team at NASA Langley, in building and testing the aircraft and associated systems, carefully calibrating the instrumentation, and carrying out the flight operations to collect the flight data used in this work, are gratefully acknowledged. Research in aircraft system identification is funded by the NASA Aviation Safety program and NASA Convergent Aeronautics Solutions (CAS) program. 


\section{References}

${ }^{1}$ Klein, V. and Morelli, E.A. Aircraft System Identification - Theory and Practice, AIAA Education Series, Reston, VA, August 2006.

${ }^{2}$ Maine, R.E. and Iliff, K.W. "Application of Parameter Estimation to Aircraft Stability and Control, The Output Error Approach,” NASA RP 1168, June 1986.

${ }^{3}$ Yang, Z., Iemura, H., Kanae, S., and Wada, K. "Identification of continuous-time systems with multiple unknown time delays by global nonlinear least-squares and instrumental variable methods,” Automatica, Vol. 43, Issue 7, July 2007, pp. 1257-1264.

${ }^{4}$ Tuch, J., Feuer, A., and Palmor, Z. J. "Time delay estimation in continuous linear time-invariant systems," IEEE Transactions on Automatic Control, Vol. 39, 1994, pp. 823-827.

${ }^{5}$ Pearson, A.E. and Wuu, C.Y. "Decoupled Delay Estimation in the Identification of Differential Delay Systems,” Automatica, Vol. 20, No. 6, 1984, pp. 761-772.

${ }^{6}$ Steers, S.T. and Iliff, K.W. "Effects of Time-Shifted Data on Flight-Determined Stability and Control Derivatives,” NASA TN D-7830, March 1975.

${ }^{7}$ X-43A Mishap Investigation Board "Report of Findings, X-43A Mishap”, Volume I, March 2002.

${ }^{8}$ Klein, V. “Aircraft Parameter Estimation in Frequency Domain,” AIAA Paper 78-1344, AIAA Atmospheric Flight Mechanics Conference, Palo Alto, CA, August 1978.

${ }^{9}$ Morelli, E.A. "High Accuracy Evaluation of the Finite Fourier Transform using Sampled Data," NASA TM 110340, 1997.

${ }^{10}$ Morelli, E.A. "Real-Time Parameter Estimation in the Frequency Domain," Journal of Guidance, Control, and Dynamics, Vol. 23, No. 5, September-October 2000, pp. 812-818.

${ }^{11}$ Morelli, E.A. "Real-Time Aerodynamic Parameter Estimation without Air Flow Angle Measurements," Journal of Aircraft, Vol. 49, No. 4, July-August 2012, pp. 1064-1074.

${ }^{12}$ Morelli, E.A. and Cooper, J., "Frequency-Domain Method for Automated Simulation Updates based on Flight Data,” AIAA-2014-0472, AIAA SciTech 2014 Conference, National Harbor, MD, January 2014.

${ }^{13}$ Jordan, Thomas L., Langford, William M., and Hill, Jeffrey S.; "Airborne Subscale Transport Aircraft Research Testbed: Aircraft Model Development”, AIAA 2005-6432, AIAA Guidance, Navigation, and Control Conference and Exhibit, San Francisco, CA, August 2005.

${ }^{14}$ Jordan, Thomas L., Foster, John V., Bailey, Roger M., and Belcastro, Christine M., "AirSTAR: A UAV Platform for Flight Dynamics and Control System Testing," AIAA-2006-3307, 25th AIAA Aerodynamic Measurement Technology and Ground Testing Conference, San Francisco, CA, June 2006.

${ }^{15}$ Cunningham, K., Foster, J.V., Morelli, E.A., and Murch, A.M., "Practical Application of a Subscale Transport Aircraft for Flight Research in Control Upset and Failure Conditions," AIAA-2008-6200, AIAA Atmospheric Flight Mechanics Conference, Honolulu, HI, August 2008.

${ }^{16}$ Murch, A. M., "A Flight Control System Architecture for the NASA AirSTAR Flight Test Facility,” AIAA 2008-6990, AIAA Guidance, Navigation, and Control Conference and Exhibit, Honolulu, HI, 2008.

${ }^{17}$ Morelli, E.A., "Practical Aspects of the Equation-Error Method for Aircraft Parameter Estimation," AIAA2006-6144, AIAA Atmospheric Flight Mechanics Conference, Keystone, CO, August 2006. 
Table 1. F-16 and T-2 Geometry and Mass Properties

\begin{tabular}{ccc}
\hline \hline & $\mathrm{F}-16$ & $\mathrm{~T}-2$ \\
\hline length $\bar{c}, \mathrm{ft}$ & 11.32 & 0.908 \\
wing span $b, \mathrm{ft}$ & 30 & 7.083 \\
wing area $S, \mathrm{ft}^{2}$ & 300 & 7.046 \\
$x_{O}$ & $0.35 \bar{c}$ & $42.628 \mathrm{in}$ \\
$y_{o}$ & 0 & $0.000 \mathrm{in}$ \\
$z_{o}$ & 0 & $0.000 \mathrm{in}$ \\
$x_{c g}$ & $0.25 \bar{c}$ & $42.728 \mathrm{in}$ \\
$y_{c g}$ & 0 & $0.000 \mathrm{in}$ \\
$z_{c g}$ & 0 & $0.519 \mathrm{in}$ \\
$m$, slug & 637.16 & 1.502 \\
$I_{x}$, slug- $\mathrm{ft}^{2}$ & 9,496 & 1.077 \\
$I_{y}$, slug- $\mathrm{ft}^{2}$ & 55,814 & 4.163 \\
$I_{z}$, slug- $\mathrm{ft}^{2}$ & 63,100 & 5.016 \\
$I_{x z}$, slug- $\mathrm{ft}^{2}$ & 982 & 0.416 \\
\hline \hline
\end{tabular}

Table 2. Frequency-Domain Equation-Error Longitudinal Parameter Estimation Results from F-16 Nonlinear Simulation Data using an Optimized Multisine Input $V_{o}=440 \mathrm{ft} / \mathrm{s}, h_{o}=10,000 \mathrm{ft}, \alpha_{o}=\theta_{o}=5 \mathrm{deg}$

\begin{tabular}{cccc}
\hline \hline & Corrected & & \\
Parameter & Time Skew Method & Data Without Time Skews & Data With Time Skews \\
$\theta$ & $\hat{\theta} \pm \hat{\sigma}$ & $\hat{\theta} \pm \hat{\sigma}$ & $\hat{\theta} \pm \hat{\sigma}$ \\
\hline$Z_{\alpha}$ & $-0.6648 \pm 0.0064$ & $-0.6670 \pm 0.0065$ & $-0.6424 \pm 0.0098$ \\
$Z_{q}$ & $-0.0703 \pm 0.0032$ & $-0.0672 \pm 0.0033$ & $-0.1103 \pm 0.0045$ \\
$Z_{\delta_{s}}$ & $-0.0014 \pm 0.0001$ & $-0.0014 \pm 0.0001$ & $-0.0010 \pm 0.0001$ \\
\hline$M_{\alpha}$ & $-3.5909 \pm 0.0384$ & $-3.6043 \pm 0.0362$ & $-3.8119 \pm 0.4706$ \\
$M_{q}$ & $-1.1032 \pm 0.0218$ & $-1.0926 \pm 0.0183$ & $-0.1448 \pm 0.2143$ \\
$M_{\delta_{s}}$ & $-0.1054 \pm 0.0006$ & $-0.1055 \pm 0.0005$ & $-0.0754 \pm 0.0062$ \\
\hline$\tau_{\alpha}$ & $0.0949 \pm 0.0030$ & - & - \\
$\tau_{\delta_{s}}$ & $-0.0996 \pm 0.0007$ & - & - \\
\hline \hline
\end{tabular}


Table 3. Frequency-Domain Equation-Error Longitudinal Parameter Estimation Results for 200 Monte Carlo Runs of F-16 Nonlinear Simulation Data using an Optimized Multisine Input $V_{o}=440 \mathrm{ft} / \mathrm{s}, h_{o}=10,000 \mathrm{ft}, \alpha_{o}=\theta_{o}=5 \mathrm{deg}$

\begin{tabular}{cccc}
\hline \hline & Corrected & & \\
Parameter & Time Skew Method & Data Without Time Skews & Data With Time Skews \\
$\theta$ & $\bar{\theta} \pm \hat{\sigma}_{\theta}$ & $\bar{\theta} \pm \hat{\sigma}_{\theta}$ & $\bar{\theta} \pm \hat{\sigma}_{\theta}$ \\
\hline$Z_{\alpha}$ & $-0.6693 \pm 0.0075$ & $-0.6686 \pm 0.0013$ & $-0.6943 \pm 0.0546$ \\
$Z_{q}$ & $-0.0704 \pm 0.0107$ & $-0.0707 \pm 0.0007$ & $-0.0608 \pm 0.0751$ \\
$Z_{\delta_{s}}$ & $-0.0013 \pm 0.0000$ & $-0.0013 \pm 0.0000$ & $-0.0013 \pm 0.0006$ \\
\hline$M_{\alpha}$ & $-3.6020 \pm 0.0425$ & $-3.5978 \pm 0.0068$ & $-4.0167 \pm 0.3761$ \\
$M_{q}$ & $-1.0868 \pm 0.0583$ & $-1.0907 \pm 0.0062$ & $-0.5346 \pm 0.9675$ \\
$M_{\delta_{s}}$ & $-0.1056 \pm 0.0003$ & $-0.1054 \pm 0.0003$ & $-0.0763 \pm 0.0343$ \\
\hline$\tau_{\alpha}$ & various \pm 0.0175 & - & - \\
$\tau_{\delta_{s}}$ & various \pm 0.0006 & - & - \\
\hline \hline
\end{tabular}

Table 4. Frequency-Domain Equation-Error Longitudinal Parameter Estimation Results for T-2 Flight Data using an Optimized Multisine Input

$$
V_{o}=130 \mathrm{ft} / \mathrm{s}, h_{o}=750 \mathrm{ft}, \alpha_{o}=\theta_{o}=4.6 \mathrm{deg}
$$

\begin{tabular}{cccc}
\hline \hline & Corrected & & \\
Parameter & Time Skew Method & Data Without Time Skews & Data With Time Skews \\
$\theta$ & $\hat{\theta} \pm \hat{\sigma}$ & $\hat{\theta} \pm \hat{\sigma}$ & $\hat{\theta} \pm \hat{\sigma}$ \\
\hline$Z_{\alpha}$ & $-2.6111 \pm 0.0331$ & $-2.6231 \pm 0.0335$ & $-2.4763 \pm 0.0336$ \\
$Z_{q}$ & $0.0793 \pm 0.0087$ & $0.0811 \pm 0.0091$ & $0.0248 \pm 0.0060$ \\
$Z_{\delta_{s}}$ & $-0.0035 \pm 0.0009$ & $-0.0029 \pm 0.0008$ & $-0.0008 \pm 0.0006$ \\
\hline$M_{\alpha}$ & $-32.394 \pm 0.840$ & $-34.812 \pm 0.669$ & $-26.337 \pm 1.519$ \\
$M_{q}$ & $-3.7275 \pm 0.2441$ & $-3.8606 \pm 0.1811$ & $-1.1885 \pm 0.2725$ \\
$M_{\delta_{s}}$ & $-0.7875 \pm 0.0238$ & $-0.7268 \pm 0.0165$ & $-0.5121 \pm 0.0291$ \\
\hline$\tau_{\alpha}$ & $0.0302 \pm 0.0038$ & - & - \\
$\tau_{\delta_{s}}$ & $-0.0750 \pm 0.0033$ & - & - \\
\hline \hline
\end{tabular}

